\title{
Dissociable memory- and response-related activity in parietal cortex during auditory spatial working memory
}

\author{
Claude Alain 1,2,3*, Dawei Shen ${ }^{1}$, He Yu' and Cheryl Grady ${ }^{1,2,3}$ \\ 1 Rotman Research Institute, Baycrest Centre for Geriatric Care, Toronto, ON, Canada \\ 2 Department of Psychology, University of Toronto, Toronto, ON, Canada \\ 3 Institute of Medical Sciences, University of Toronto, Toronto, ON, Canada
}

Edited by:

Pascal Belin, University of Glasgow, UK

Reviewed by:

István Winkler, University of Szeged

Hungary

Neil M. Mclachlan, University of

Melbourne, Australia

${ }^{*}$ Correspondence:

Claude Alain, Rotman Research

Institute, Baycrest Centre for Geriatric

Care, 3560 Bathurst Street, Toronto,

ON, Canada M6A $2 E 1$.

e-mail: calain@rotman-baycrest.on.ca
Attending and responding to sound location generates increased activity in parietal cortex which may index auditory spatial working memory and/or goal-directed action. Here, we used an $n$-back task (Experiment 1) and an adaptation paradigm (Experiment 2) to distinguish memory-related activity from that associated with goal-directed action. In Experiment 1, participants indicated, in separate blocks of trials, whether the incoming stimulus was presented at the same location as in the previous trial (1-back) or two trials ago (2-back). Prior to a block of trials, participants were told to use their left or right index finger. Accuracy and reaction times were worse for the 2-back than for the 1-back condition. The analysis of functional magnetic resonance imaging data revealed greater sustained task-related activity in the inferior parietal lobule (IPL) and superior frontal sulcus during 2-back than 1-back after accounting for response-related activity elicited by the targets. Target detection and response execution were also associated with enhanced activity in the IPL bilaterally, though the activation was anterior to that associated with sustained task-related activity. In Experiment 2, we used an event-related design in which participants listened (no response required) to trials that comprised four sounds presented either at the same location or at four different locations. We found larger IPL activation for changes in sound location than for sounds presented at the same location. The IPL activation overlapped with that observed during the auditory spatial working memory task. Together, these results provide converging evidence supporting the role of parietal cortex in auditory spatial working memory which can be dissociated from response selection and execution.

Keywords: attention, auditory, fMRI, parietal cortex, spatial, dorsal stream, motor response, goal-directed action

\section{INTRODUCTION}

Evidence from neuroanatomical and neurophysiological studies in non-human primates (e.g., Romanski et al., 1999; Poremba et al., 2003) as well as neuroimaging studies in humans (e.g., Alain et al., 2001; Maeder et al., 2001) suggest that auditory cognition depends on at least two functionally distinct pathways. A ventral pathway thought to play an important role in identifying the incoming sound (i.e., "what") and a more dorsal pathway associated with "where" the sound is spatially located (Rauschecker, 1998). While the ventral stream continues to be associated with the identification and/or categorical encoding of sound objects (McLachlan and Wilson, 2010), there is considerable debate regarding the functional role of the dorsal (where) processing stream in audition. Some authors have proposed that the dorsal pathway supports processes involved in localizing and remembering sound location (e.g., Alain et al., 2001, 2008; Maeder et al., 2001; Arnott et al., 2004, 2005; Rama et al., 2004; Deouell et al., 2007) as well as other sound attributes that could be spatially encoded in sensory memory such as pitch, motion, and loudness (McLachlan and Wilson, 2010). Others have recast the dorsal stream as a pathway critical for sensory-motor integration and goal-directed action (Hickok and Poeppel, 2000; Zatorre et al., 2002; Warren et al., 2005) akin to the perceptionaction model of visual scene analysis (Goodale, 2008).
These theoretical accounts are not mutually exclusive and suggest that multiple functions may be supported by the dorsal streams during auditory scene analysis. Indeed, evidence from animal studies has shown that the parietal cortex plays an important role in spatial representation, spatial attention, motor planning, sensorymotor integration, and goal-directed action (for reviews, Andersen et al., 1997; Cohen, 2009). Hence, different regions within the parietal cortex may support sound object location and auditory-motor transformation for goal-directed action. In a recent study, Alain et al. (2008) used a mixed-block event-related functional magnetic resonance imaging (fMRI) design in which participants responded to occasional repetitions in either sound location or semantic category. The analysis of fMRI data revealed greater activity in right dorsal brain regions, including the inferior parietal lobule (IPL) and superior frontal sulcus, during the location than during the category task, after accounting for response-related activity. Responding to infrequent targets was associated with enhanced signal in the left pre- and post-central gyrus, prefrontal cortex and bilateral IPL. These results are consistent with a dual role for the parietal cortex in auditory scene analysis - one involved in representation of auditory space and another that underlies auditory motor transformation (Alain et al., 2008). However, as neither working memory load nor response demand were manipulated in that study, it remains unclear 
whether similar regions support memory- and goal-directed action or whether auditory spatial memory and motor transformation can be dissociated within the parietal cortex.

The present study aims to further test this dual role of the parietal cortex during auditory scene analysis by directly manipulating stimulus-response mapping (i.e., left vs. right index finger) and working memory load during an $n$-back task (Experiment 1 ). Our hypothesis is that, within the parietal cortex, memory-related activity for sound location, as measured by fMRI, can be dissociated from activity related to sensory-motor integration and goal-directed action. To test this hypothesis, we used a mixed-block event-related design that allows the separation of sustained task-related activity that occurs throughout a block of trials from response-related activity elicited by infrequent target stimuli. Participants performed an $n$-back task that required pressing a button to infrequent repetition in sound location (i.e., target). The areas within the parietal cortex that play an important role in auditory spatial working memory should be modulated by working memory load whereas those indexing stimulus-response mapping and goal-directed action should vary as a function of response demand. In Alain et al. (2008) as well as in Experiment 1, it remains possible that maintaining the stimulus-response mappings and task goals could also produce sustained fMRI activity in parietal cortex. If the IPL plays an important role in processing sound location per se then one should observe IPL activation to variations in sound location during passive listening without any overt motor response required. In Experiment 2, we used an adaptation paradigm to examine memory-related activity in the absence of stimulus-response mapping (i.e., when no response was required). In that experiment, sounds from various semantic categories were presented at either the same or different locations. Our hypothesis was that changes in sound location would generate greater activation in parietal cortex than repetition of sound location.

\section{MATERIALS AND METHODS PARTICIPANTS}

Twenty-eight participants provided written informed consent to participate in the study according to the University of Toronto and Baycrest Hospital Human Subject Review Committee guidelines. In Experiment 1, there were seven women and five men aged between 21 and 30 years $($ mean $=26.33, \mathrm{SD}=2.90)$. In Experiment 2 , there were eight women and eight men aged between 19 and 31 years ( mean $=25.31, \mathrm{SD}=3.86$ ). All participants were right-handed and had pure tone amplitude thresholds within normal limits for octave frequencies from 250 to $8000 \mathrm{~Hz}$ (both ears).

\section{STIMULI AND TASK \\ Experiment 1}

Stimuli consisted of meaningful sounds from three semantic categories: animal (e.g., dog bark, bird chirp), human non-speech sounds (e.g., cough, laugh), and musical instruments (e.g., flute, clarinet). In each category, 10 different exemplars were chosen from a larger databank and only those that could be unambiguously categorized as animal, human, or musical sounds were included in the study. All auditory stimuli were edited to have durations of $1005 \mathrm{~ms}$. Onsets and offsets were shaped by first and second halves of an 8-ms Kaiser window, respectively. Stimuli were digitally generated with a 16-bit resolution and a $12.21-\mathrm{kHz}$ sampling rate, passed through a digital-to-analog RP2 converter (Tucker-Davis Technology, Gainesville, FL, USA). They were delivered to the listener at about $88 \mathrm{~dB}$ sound pressure level (root mean square) by means of circumaural, fMRI-compatible headphones (Avotec, Jensen Beach, FL, USA), acoustically padded to suppress scanner noise by $25 \mathrm{~dB}$. Stimuli were presented at three possible azimuth locations relative to straight ahead $\left(-90^{\circ}, 0^{\circ},+90^{\circ}\right)$ using head-related transfer functions (HRTF) that replicated the acoustic effects of the head and ears of an average listener (Wenzel et al., 1993).

Participants performed a 1-back and 2-back working memory (WM) task where sound location was occasionally repeated. Within a block of trials, 20 sounds were presented including four, five, or six target sounds (i.e., location repeat). The stimulus onset interval was $2 \mathrm{~s}$ and the inter-target interval varied between 4 and $12 \mathrm{~s}$ ( $2 \mathrm{~s}$ steps, rectangular distribution). Participants were instructed to press a button as quickly as possible using their index finger only when a sound location was repeated. Participants' responses were registered using an fMRI-compatible response pad (Lightwave Technologies, Surrey, BC, Canada). Prior to a block of trials, participants were presented with a visual word prompt (e.g., 1-back left) on a screen indicating which task they should perform (1-back or 2-back) and which index finger (left or right) they should use to make their response. This prompt appeared on the screen $6 \mathrm{~s}$ prior to the first sound and remained on the screen for $4 \mathrm{~s}$. For instance, when the word "1-back left" was presented participants were required to press a button as quickly as possible with their left index finger whenever the incoming stimulus occurred at the same location as the one before regardless of changes in, or repetition of, sound category. Stimuli were presented in pseudo-random draw from the same set of stimuli with each sound category and sound location presented with equal probability. Aside from the prompt, the set of stimuli used was identical in all four conditions.

Participants were given the opportunity to practice the task prior to the fMRI experiment. Those who failed to obtain at least $75 \%$ correct in the 1-back task were thanked for their time and did not participate in the fMRI study. During the fMRI experiment, participants performed each designated task (e.g., 1-back left) for $40 \mathrm{~s}$ followed by a 26 -s rest period in which no stimuli were presented. This on/off sequence was repeated six times in each scan for a total duration of $5 \mathrm{~min}$ and $10 \mathrm{~s}$ and each participant took part in six fMRI scans. The tasks alternated throughout the fMRI run and the order of the tasks was counterbalanced across fMRI scans and participants. Participants kept their eyes open throughout all scans.

\section{Experiment 2}

Stimuli for Experiment 2 consisted of meaningful sounds from four categories: human non-speech sounds (e.g., laughter), animal sounds (e.g., a rooster crowing), musical sounds (e.g., cello), and machine noise (e.g., road construction). Each sound could be presented in one of four locations: $-95^{\circ},-30^{\circ},+30^{\circ},+95^{\circ}$. Each trial consisted of four sounds, presented for $1005 \mathrm{~ms}$ each with a $295-\mathrm{ms}$ interval between each sound. The inter-trial interval randomly varied between 4 and $8 \mathrm{~s}$ ( $1 \mathrm{~s}$ step, rectangular distribution).

The trials could be one of four condition types: same sound, same location $\left(\mathrm{S}_{S} \mathrm{~S}_{\mathrm{L}}\right)$; same sound, different location $\left(\mathrm{S}_{S} \mathrm{D}_{\mathrm{L}}\right)$; different sound, same location $\left(D_{S} S_{L}\right)$; different sound, different location 
$\left(D_{S} D_{L}\right)$. For instance, in $D_{S} S_{L}$ and $D_{S} D_{L}$ trials, the four different sounds were from different categories whereas in $S_{S} D_{L}$ and $D_{S} D_{L}$ trials, the stimuli were presented at four different locations. In the $S_{S} S_{L}$ and $S_{S} D_{L}$ trials, the same sound was repeated four times. However, we use sounds from different categories between trials to insure that the results are not specific to a particular sound type. During the fMRI acquisition, the order of trials was constrained randomly (i.e., no two identical trials in a row). The stimulus intensity and delivery was identical to that of Experiment 1. Also, as in Experiment 1, we used HRTF to present sounds at the four virtual locations along the azimuth. Participants were administered five runs in total (lasting $350 \mathrm{~s}$ each), and in each scanning run there were five trials of each condition. A given sound was used in only one trial, so that each trial across the five runs used unique stimuli. Participants were told to listen to the auditory stimuli and to keep their eyes open throughout the scanning runs.

\section{fMRI SCANNING AND DATA ANALYSIS Experiment 1}

Participants were scanned using a research-dedicated whole-body 3.0 T MRI system (Siemens Tim Trio - 3T software level Syngo MR 2006 VB13T) with a standard quadrature bird-cage head coil. Each scan sequence began with a 20 -s period where no stimuli were presented, followed by six alternations between task and rest periods, the latter serving as a baseline.

Functional imaging was performed to measure brain activation by means of the blood oxygenation level-dependent (BOLD) effect (Ogawa et al., 1990). Functional data were acquired using a whole head T2*-weighted echo-planar image (EPI) sequence (TE: $30 \mathrm{~ms}$; TR: 2 s; flip angle: $70^{\circ}$; 30 oblique axial slices with interleaved acquisition, $3.125 \mathrm{~mm} \times 3.125 \mathrm{~mm} \times 5 \mathrm{~mm}$ voxel resolution; FOV: $20 \mathrm{~cm}$; AM: $64 \times 64)$. Physiological respiratory and cardiac waveforms were recorded from the bellows and photoplethysmograph peripherals on the scanner, respectively, using LabView (National Instruments, Austin, TX, USA). The RETROICOR technique (Glover et al., 2000) was subsequently used to remove these structured noise sources from fMRI time-series data.

Data preprocessing and analyses were performed using Analysis of Functional Neuroimages software (AFNI version 2.56a; (Cox, 1996). In each run, the first 10 scans were discarded to allow the magnetization to reach steady state. In the preprocessing stage, time-series data were spatially co-registered to correct for head motion using a 3D Fourier transform interpolation. For each run, images acquired at each point in the series were aligned volumetrically, using the 3 dvolreg plugin for AFNI, to a reference image acquired during the scanning session. The alignment parameters were computed by an iterative weighted least squares fit to the reference images. The peak range of head motion was less than $1.5 \mathrm{~mm}$ for all participants. The co-registration results were also checked visually for additional quality control. Lastly, the images were de-trended by means of $3 \mathrm{dDeconvolve}$ from AFNI using a linear fitting.

We analyzed the fMRI data with the general linear model (GLM) using separate regressors for representing the sustained and transient activity (Experiment 1). This approach allowed us to dissociate sustained task-related activity occurring throughout the block of trials from transient target-related activity elicited by infrequent repetition of sound location (Visscher et al., 2003; Dosenbach et al., 2006; Scheibe et al., 2006; Alain et al., 2008). For the sustained task-related effect, the shape of the response was modeled as a "gamma" function convolved with a boxcar function of width equal to the duration of the block. For the responserelated effect, we also used a gamma function time-locked on targets (i.e., 1-back or 2-back). Only trials where participants responded correctly were included in the event-related analysis. The activation maps created by the GLMs for each condition and each participant were then transformed into stereotaxic space and spatially smoothed with a Gaussian filter with a full width at half maximum (FWHM) value of $6.0 \mathrm{~mm}$. These last two steps were performed to facilitate the subsequent group analysis which consisted of a voxel-wise, mixed effects (conditions fixed, participants random) $2 \times 2$ ANOVA with response mode (left vs. right index finger response) and working memory load (1-back vs. 2-back) as within-subjects factors.

A spatial cluster extent threshold was used to correct for multiple comparisons using AlphaSim with 1000 MonteCarlo simulations taking into account the entire spiral matrix. Using an uncorrected $p$ value threshold of 0.001 , this procedure yielded a minimum cluster size of $196 \mu$ (four voxels in the original acquisition space) with a map-wise false-positive probability of $p<0.025$. Only significant activations that had a cluster size of $196 \mu \mathrm{l}$ (radius of $2 \mathrm{~mm}$ ) or greater are reported.

\section{Experiment 2}

The data acquisition and analysis were identical to that of Experiment 1 with the following exceptions. For the anatomical scan, we used axial slices (not oblique axial). For the functional data, we acquired 28 axial slices rather than 30 oblique axial slices. Experiment 2 used an event-related design, for which each event was modeled as a "gamma" function time-locked on sound onset. The activation maps created by the GLMs for each condition and each participant were then transformed into stereotaxic space and spatially smoothed with a Gaussian filter with a FWHM value of $6.0 \mathrm{~mm}$. These last two steps were performed to facilitate the subsequent group analysis which consisted of a voxel-wise, mixed effects (conditions fixed, participants random) $2 \times 2$ ANOVA with stimulus type (sound identity vs. sound location) and conditions (change vs. repeat) as within-subjects factors. This analysis allowed us to examine the main effect of condition on sound location $\left(S_{S} S_{L}+D_{S} S_{L}\right.$ vs. $\left.S_{S} D_{L}+D_{S} D_{L}\right)$ and sound identity $\left(S_{S} S_{L}+S_{S} D_{L}\right.$ vs. $\left.D_{S} S_{L}+D_{S} D_{L}\right)$ separately as well as their possible interaction.

\section{RESULTS EXPERIMENT 1 \\ Behavior}

The group mean accuracy and response time are presented in Table 1. The hit rate was lower in the 2 -back $($ mean $=82, \mathrm{SE}=3 \%)$ than in the 1 -back $($ mean $=92, \mathrm{SE}=2 \%$ ) condition, $F(1,11)=18.96$, $p<0.01$. Participants were also slower in the 2 -back (mean $=902 \mathrm{~ms}$, $\mathrm{SE}=54 \mathrm{~ms}$ ) than 1 -back (mean $=794 \mathrm{~ms}, \mathrm{SE}=36 \mathrm{~ms})$ condition, $F(1,11)=8.75, p<0.05$. They also made slightly more false alarms during the 2 -back $($ mean $=7 \%, \mathrm{SE}=2 \%)$ than 1 -back $($ mean $=5 \%$, $\mathrm{SE}=2 \%$ ) condition, although the difference did not reach significance, $F(1,11)=2.49, p=0.14$. The main effect of response mode 
on hit rate and response time was not significant $[F(1,11)=0.15$ and 2.08 , respectively] nor was the interaction between working memory load and response mode.

\section{fMRI results}

Figure 1 shows the results of the mixed model analysis used to separate sustained task-related and transient target-related activity. This analysis reveals distinct brain regions supporting sustained task-related (i.e., not related to response) and transient target-related activity (i.e., activity related to responding). Sustained task-related activity was characterized by enhanced activity in bilateral regions of the superior temporal gyrus ${ }^{1}$, the IPL, the inferior, middle, and superior frontal gyri. In comparison, transient target-related activity was characterized by enhanced activity in temporo-parietal junction and IPL bilaterally, as well as pre- and post-central gyri. More importantly, within the IPL, target detection and response execution generated an increased fMRI signal that was anterior and inferior to

${ }^{1}$ Infrequent sound repetition (i.e., target) in Experiment 1 did not yield changes in activation that could be distinguished from the sustained activity. This could partly be due to reduced neural activity associated with repeating sound location (i.e., target).

Table 1 | Group mean accuracy (hits and false alarms, FAs) and response time as a function of working memory and response mode.

\begin{tabular}{lccc}
\hline & Hits (\%) & FAs (\%) & $\begin{array}{l}\text { Response } \\
\text { time (ms) }\end{array}$ \\
\hline \multicolumn{2}{l}{ LEFT INDEX FINGER } & & \\
1-back & $92(1.9)$ & $5.6(1.7)$ & $817(35)$ \\
2-back & $81(3.6)$ & $6.5(1.6)$ & $899(49)$ \\
RIGHT INDEX FINGER & & \\
1-back & $92(2.7)$ & $5.1(1.6)$ & $771(39)$ \\
2-back & $83(2.8)$ & $7.1(2.0)$ & $906(61)$ \\
\hline
\end{tabular}

Standard error of the mean is indicated in the parenthesis. that observed for sustained activity during auditory spatial working memory. In the following analyses, we examined the impact of working memory load and response mode on the sustained task-related and transient target-related activity observed in the IPL.

Sustained task-related activity during auditory spatial working memory. Figure 2A shows the difference in sustained task-related activity between 1- and 2-back conditions. Relative to 1-back, increased working memory load for sound location was associated with enhanced BOLD signal in IPL bilaterally, right superior parietal cortex, right middle temporal gyrus, insula bilaterally, left inferior frontal gyrus, left middle frontal gyrus, and left superior frontal gyrus (see Table 2). In addition to enhanced activity in several dorsal brain regions, increased auditory spatial working memory load was accompanied by reduced BOLD effects in ventral areas (not shown), thought to play an important role in working memory for sound identity, including the anterior portion of the superior temporal gyrus, inferior frontal gyrus, and medial temporal lobe (i.e., parahippocampal cortex bilaterally). The main effect of response mode on sustained taskrelated activity was not significant nor was the interaction between working memory load and response mode. More importantly, the sustained task-related increased activity in inferior and superior parietal cortex was little affected by varying the response mode (Figure 2B). That is, maintaining a particular stimulus-response mapping (i.e., responding with the left or right index finger) did not yield reliable differences in the parietal cortices.

Transient target-related activity. The effects of response mode and auditory spatial working memory load on transient target-related activity are shown in Figure 3. As expected, there was a transient enhanced BOLD signal in pre- and post-central gyrus contralateral to the hand used to respond during the task. Responding with the non-dominant (left) hand also yielded greater activity in the left cerebellum, right thalamus, and right superior parietal cortex compared to when participants were using their right hand (Table 3).

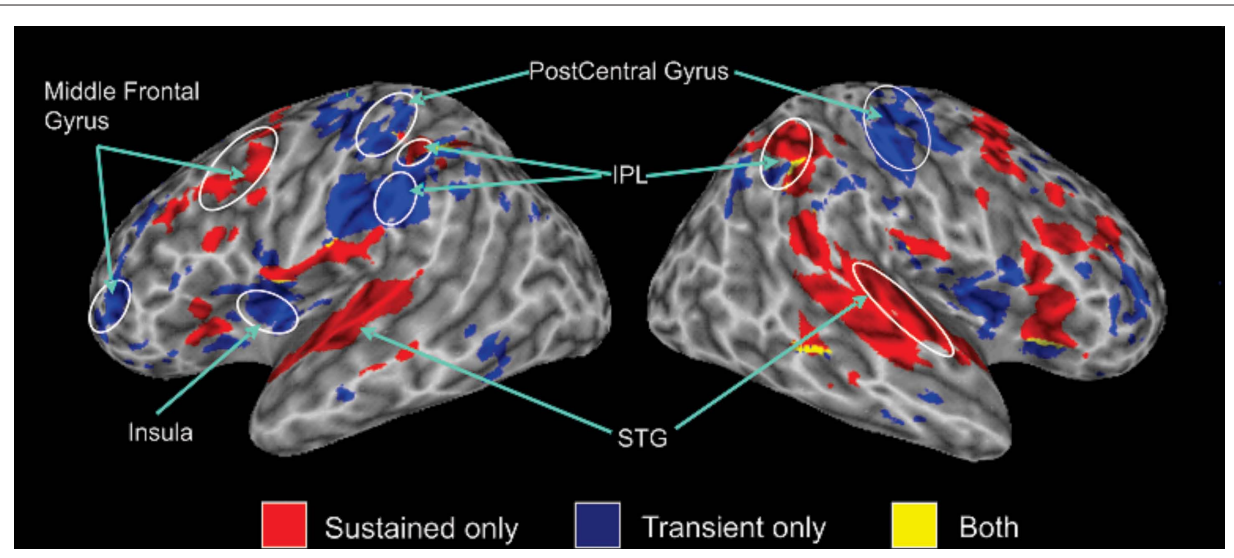

FIGURE 1 | 3-D view revealing sustained and transient activity relative to rest (no sound presented) on the average structural image of all participants. In this and subsequent figures, all activations entered in the conjunction analysis had cluster activations that contained at least $196 \mu \mathrm{l}$ and were significant at $p<0.05$ corrected. The conjunction analysis reveals commonalities in activation (overlapping regions) between experimental conditions. The blue color indicates transient response-related activity averaged over both response conditions, the areas in red color correspond to sustained task-related activity, and yellow color show the areas of overlap between transient and sustained activity. Activation maps in this figure and subsequent figures are displayed on the cortical surface using surface mapping (SUMA, Argall et al., 2006). IPL, inferior parietal lobule; STG, superior temporal gyrus. 


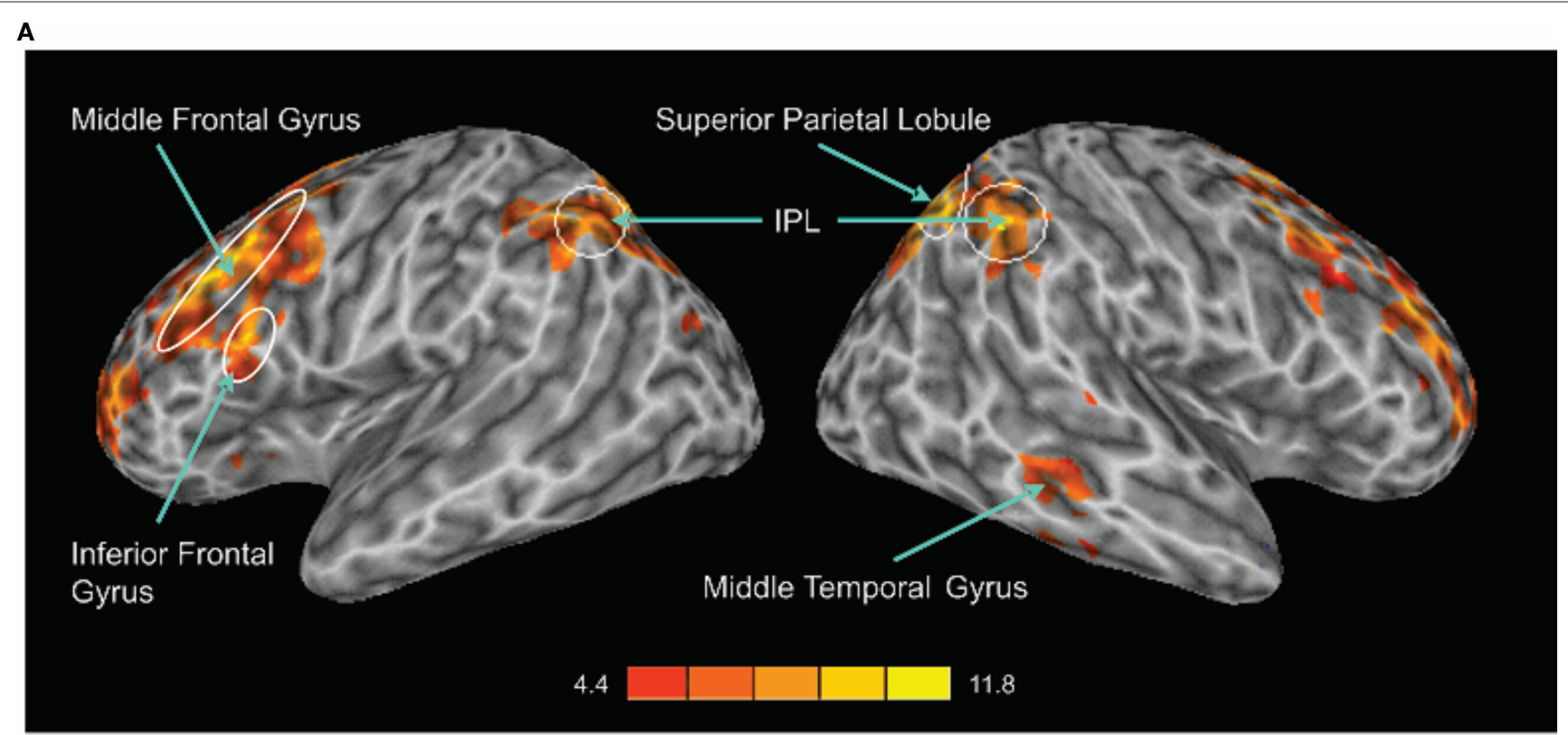

B

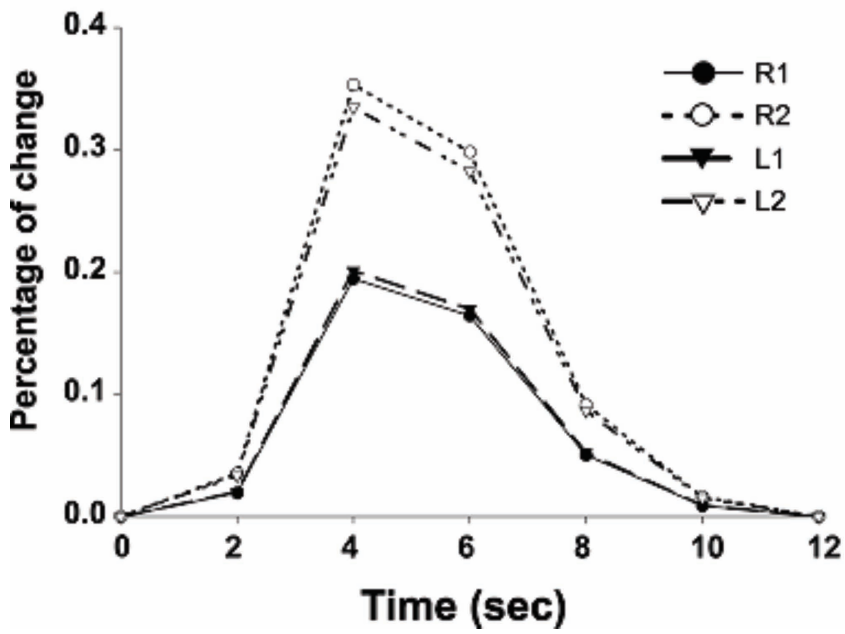

FIGURE 2 | (A) Effects of auditory spatial working memory load on BOLD response (i.e., 2-back vs. 1-back). The enhanced activity revealed true increases and does not reflect reduced activity in 1-back relative to baseline. (B) Time course of the BOLD effect in the inferior parietal lobule as a function of memory load and response mode. Each trace reflects the average activity based on nine

\section{Left Hemisphere}

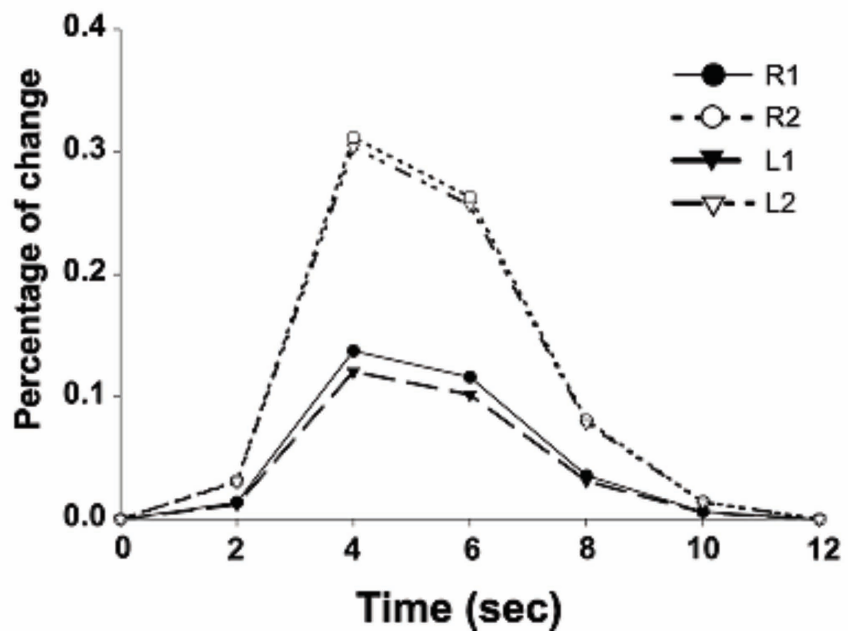

voxels (centered on a voxel with highest intensity) in the IPL region. Note, the increased activity with increasing memory load and the similarity in activity while responding with the left or right index finger. IPL, inferior parietal lobule; $L 1$, left hand 1-back; L2, left hand 2-back; R1, right hand 1-back; R2, right hand 2-back.
The transient enhanced response-related activity in motor areas was little affected by increasing working memory load. In the right IPL, there was a small area that showed an interaction between working memory load and response mode. That is, the enhanced transient target-related activity in IPL was present only when the participants used their left index finger during the 2-back task. The peak activation in the IPL for the transient target-related activity $(x=44, y=-38, z=55)$ was superior and anterior to that observed for sustained task-related activity $(x=44, y=-50, z=42)$.

In summary, distinct areas within the IPL show sensitivity to variation in auditory spatial working memory load and response mode. Our findings replicate and extend earlier studies (e.g., Rama 
Table 2 | Enhanced sustained activity during 2-back vs. 1-back working memory for sound location.

\begin{tabular}{|c|c|c|c|c|c|c|c|}
\hline \multirow[b]{2}{*}{ Brain regions } & \multicolumn{4}{|c|}{ Peak Talairach co-ordinate } & \multicolumn{3}{|c|}{$t$-values } \\
\hline & BA & $x$ & $y$ & $z$ & 2 vs. 1 & 2 vs. B & 1 vs. $B$ \\
\hline L middle frontal gyrus & 6 & -28 & -2 & 59 & 9.01 & 8.99 & 2.94 \\
\hline$L$ inferior frontal gyrus & 9 & -42 & 4 & 29 & 5.23 & 6.23 & 5.44 \\
\hline L insula & 13 & -31 & 21 & 12 & 4.40 & 8.01 & 5.49 \\
\hline L supramarginal gyrus & 40 & -39 & -39 & 36 & 7.34 & 8.49 & 4.19 \\
\hline $\mathrm{L}$ cerebellum & & -28 & -59 & -43 & 5.17 & 5.60 & 2.34 \\
\hline R superior frontal gyrus & 6 & 4 & 14 & 49 & 6.88 & 7.41 & 4.18 \\
\hline R middle frontal gyrus & 6 & 35 & 0 & 55 & 5.32 & 5.95 & 2.72 \\
\hline$R$ insula & 13 & 35 & 20 & 13 & 5.68 & 10.61 & 7.24 \\
\hline $\mathrm{R}$ middle temporal gyrus & 21 & 62 & -34 & -6 & 4.73 & 6.10 & 1.49 \\
\hline
\end{tabular}

All activations are $>196 \mu$ land significant at $p<0.05$ corrected.

BA, Brodmann's area; 2 vs. 1, 2-back versus 1-back contrast; 2 vs. B, 2-back versus baseline; 1 vs. B, 1-back versus baseline.

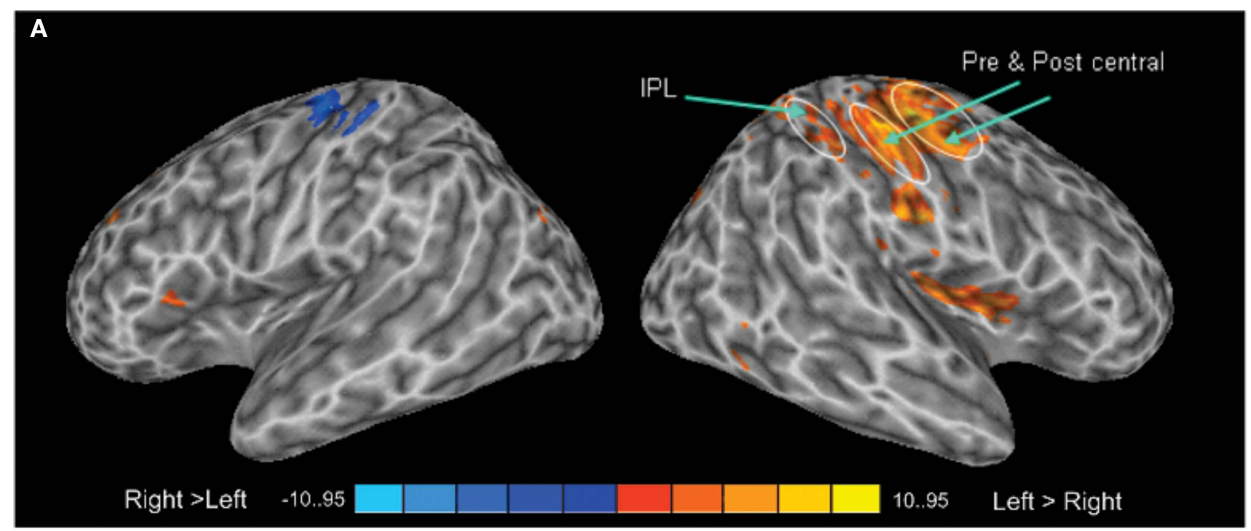

B

Right Precentral Gyrus

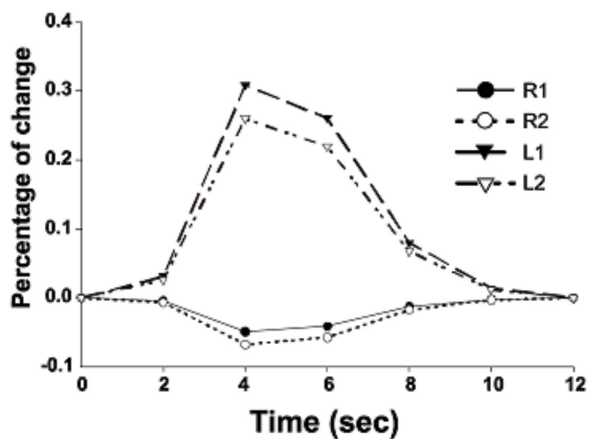

FIGURE 3 | (A) Activation maps reveal differences in target-related activity as a function of response mode. The blue color indicates transient response-related activity for the right index finger whereas the areas in red color correspond to enhanced activity associated with responding with the left index finger. (B) Time course of the BOLD effect in the right pre-central gyrus and right inferior parietal

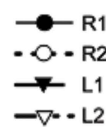

$-\nabla \cdot \cdot 12$

Right IPL

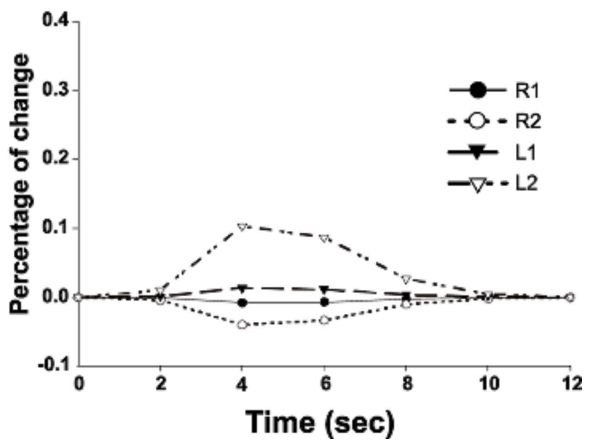

lobule (IPL) as a function of memory load and response mode. Each trace reflects the average activity based on nine voxels (centered on a voxel with highest intensity) in the right pre-central gyrus and right IPL region. Note the increased activity in the right IPL with increasing memory load while responding with the left index finger. L1, left hand 1-back; L2, left hand 2-back; R1, right hand 1-back; R2, right hand 2-back. 


\begin{tabular}{|c|c|c|c|c|c|c|c|}
\hline \multirow[b]{2}{*}{ Brain regions } & \multicolumn{4}{|c|}{ PeakTalairach co-ordinate } & \multicolumn{3}{|c|}{$t$-values } \\
\hline & BA & $x$ & $y$ & $z$ & L vs. R & L vs. B & R vs. B \\
\hline \multicolumn{8}{|l|}{ LEFT > RIGHT } \\
\hline L cingulate & 32 & -2 & -8 & 41 & 4.42 & 3.78 & 2.38 \\
\hline L superior frontal gyrus & 8 & -38 & 18 & 52 & 4.07 & 4.12 & 0.31 \\
\hline L cerebellum & & -20 & -43 & -21 & 5.94 & 6.19 & 1.02 \\
\hline L cerebellum & & -20 & -49 & -50 & 5.60 & 4.53 & -0.75 \\
\hline L cerebellar tonsil & & -23 & -26 & -37 & 5.90 & 4.40 & -1.03 \\
\hline$R$ cingulate & 24 & 5 & -2 & 41 & 4.00 & 4.13 & 1.87 \\
\hline R pre-central gyrus & 4 & 34 & -23 & 61 & 6.09 & 5.19 & -1.79 \\
\hline R post-central gyrus & 3 & 43 & -23 & 54 & 7.61 & 6.34 & -2.37 \\
\hline$R$ insula & 13 & 36 & -9 & 14 & 4.94 & 5.08 & 0.05 \\
\hline $\mathrm{R}$ thalamus & & 17 & -13 & 8 & 4.59 & 4.54 & 2.02 \\
\hline R superior parietal cortex & 7 & 29 & -53 & 65 & 5.10 & 3.69 & 0.22 \\
\hline R precuneus & 7 & 4 & -36 & 47 & 5.03 & 4.34 & -0.68 \\
\hline \multicolumn{8}{|l|}{ RIGHT > LEFT } \\
\hline L pre-central gyrus & 4 & -37 & -24 & 54 & 6.62 & 0.30 & 8.07 \\
\hline L post-central gyrus & 3 & -39 & -24 & 55 & 7.17 & 0.56 & 6.79 \\
\hline
\end{tabular}

All activations are $>196 \mu$ land significant at $p<0.05$ corrected.

$B A$, Brodmann's area; $L$ vs. $R$, left versus right index finger; $L$ vs. $B$, left versus baseline; $R$ vs. $B$, right versus baseline.

et al., 2004; Alain et al., 2008) by showing IPL activity that covaries with working memory load for sound location independently of overt motor responses. This sustained task-related activity may index location and/or memory-related processes. However, it could also reflect the maintenance of stimulus-response mappings and task goals which must be retained throughout the task. If the IPL plays an important role in processing sound location per $s e$ then one should observe IPL activation to variations in sound location during passive listening without any overt motor response required. In Experiment 2, we used a variant of the adaptation paradigm to further investigate the brain areas involved in the representation of sound location. The adaptation paradigm is based on the property that neuronal populations reduce their firing rate with repeated stimulation and have been successfully employed to investigate the representation of visual objects (e.g., Buckner et al., 1998) and speaker identity (Belin and Zatorre, 2003).

\section{EXPERIMENT 2}

\section{fMRI results}

Relative to silent baseline, all trial types generated enhanced activity in auditory cortex along the superior temporal plane that extended from the anterior and posterior portion of the superior temporal gyrus. To isolate brain areas that show release from adaptation (i.e., greater activity when a sound or its location differs relative to when it is repeated) for sound identity and sound location, we performed a $2 \times 2$ ANOVA with stimulus type (sound identity vs. sound location) and condition (change vs. repeat) as factors. The main effect of condition was significant for sound identity and sound location (Figure 4). For sound identity, this analysis reveals greater activation in the superior temporal gyrus bilaterally that extended in the anterior portion of the temporal lobe when sound identity changed compare to when the same sound was repeated. Changes in sound identity also yielded an increased BOLD response in the left middle temporal gyrus. There was no reliable difference in parietal activity when the same sound was repeated vs. when it changed. For sound location, we found greater BOLD responses in IPL bilaterally when the location changed than when it was repeated. The region within the IPL that showed sensitivity to adaptation for sound location (i.e., Experiment 2) overlapped with the IPL region that was modulated by auditory spatial working memory load (i.e., Experiment 1, see Figure 5). There was no reliable difference in activity in the superior temporal gyrus when sounds were presented at the same vs. different locations. The interaction between stimulus type and condition was also significant. This interaction indicated greater changes in activity in parietal cortex for changes in sound location when the sound identity repeated than when both sound identity and sound location changed.

\section{DISCUSSION}

The present study was designed to assess sensitivity in the IPL to auditory spatial working memory load and stimulus-response mapping. We found a distinct pattern of activation for processing and remembering sound location which differed from response-related activity associated with target detection. The lack of interaction between working memory and response mode reveals a dual role of IPL during auditory scene analysis: one related to the monitoring and updating of sound location in working memory and one related to decision making processes, response selection, and action. This interpretation is compatible with the most recent incarnation of the dual-pathway model (Rauschecker and Scott, 2009) which also posits multiple functions within the dorsal streams to account for speech perception and production. This functional segregation also underscores the cytoarchitectonically defined subdivisions within 


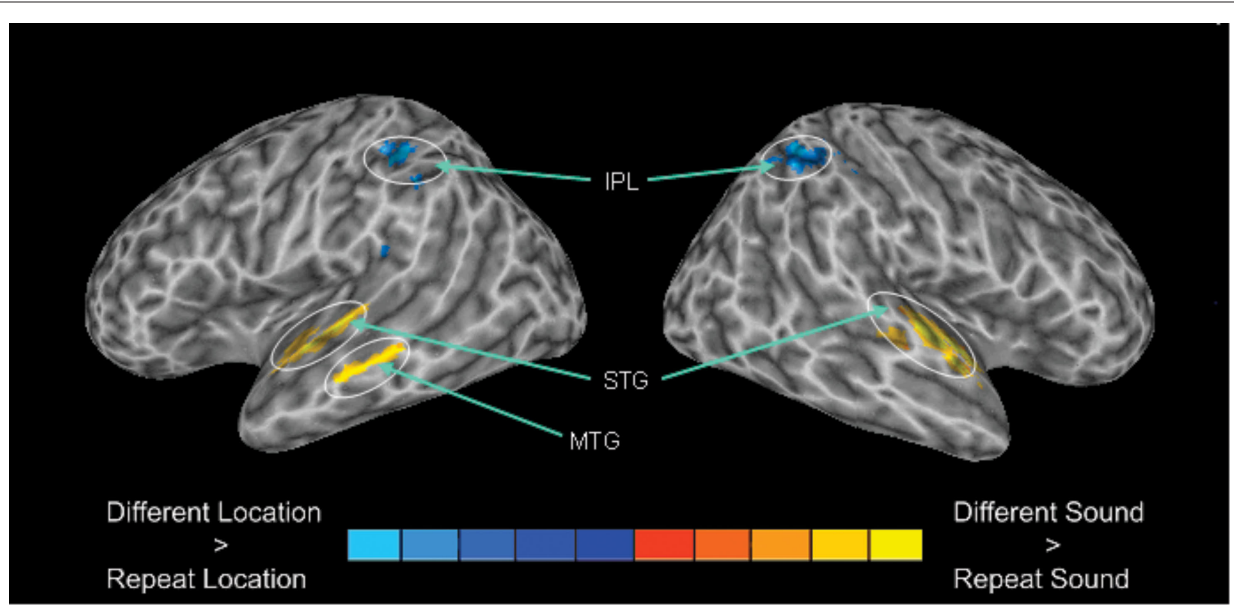

FIGURE 4 | 3-D view revealing enhanced activation for changes in sound category $\left(D_{S} S_{L}\right)$ vs. changes in sound location ( $\left.S_{S} D_{L}\right)$. The blue color indicates greater activity for changes in sound identity whereas the areas in red corresponds to changes in sound location. IPL, inferior parietal lobule; STG, superior temporal gyrus; MTG, media temporal gyrus.

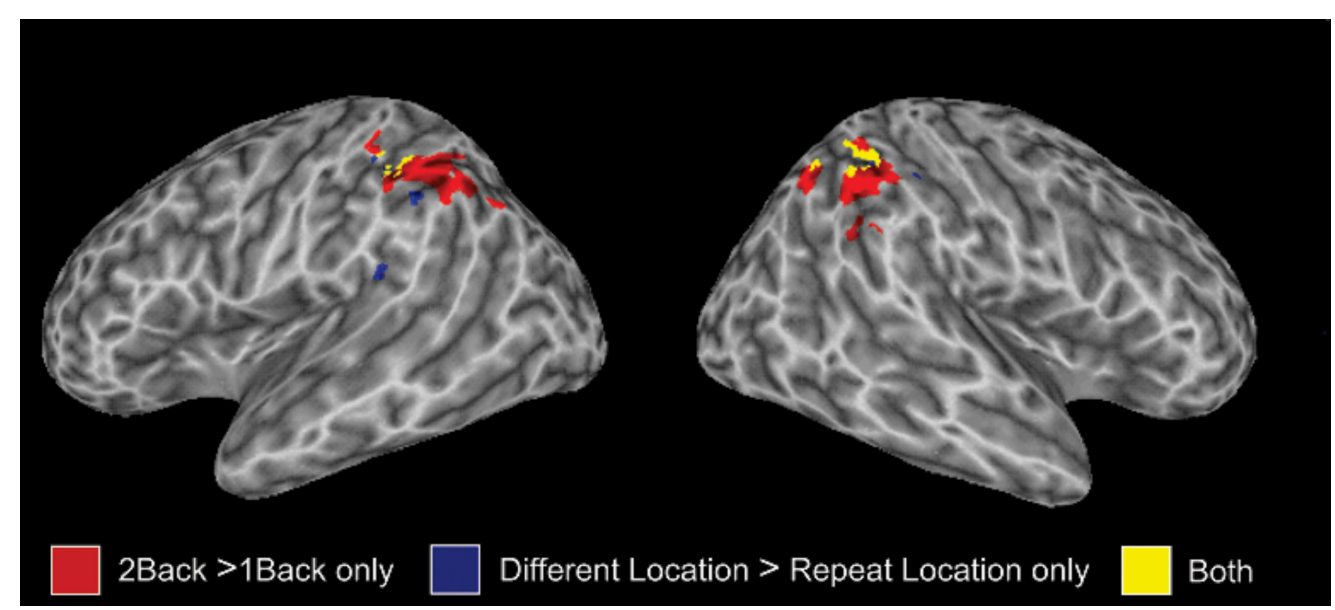

FIGURE 5|3-D view showing the degree of overlap in memory-related activity in inferior parietal lobule (IPL) from Experiment 1 and Experiment 2. The effect of working memory load is shown in red whereas the changes in parietal activity with variation in sound location are shown in blue. The overlap is shown in yellow.

IPL (Uddin et al., 2010), which may support distinct functions during auditory scene analysis. Hence, it appears that during auditory scene analysis the dorsal pathway can be divided into at least two "streamlets" that support representation of auditory space and stimulus-response mapping, respectively.

The load-related activity in IPL and superior frontal gyrus is similar to that observed in a previous study (Martinkauppi et al., 2000) and provides further support for the memory account of the dorsal stream (e.g., Alain et al., 2001, 2008; Rama et al., 2004; Arnott et al., 2005). More importantly, these load-related changes in brain activity for sound location remained even after controlling for transient target-related activity and motor response. Our results extend those from our previous study (Alain et al., 2008) by showing robust sustained activity in IPL that varied with spatial working memory load independently of changes in stimulus-response mapping. That is, varying response mode has little impact on memory-related activity in IPL.
In the present study, the IPL region modulated by spatial working memory load was also sensitive to changes in sound location even when there was no response required from the participants. The changes in IPL activity during passive listening argue against a strict sensorymotor account of the dorsal stream. The location-specific adaptation in IPL is a surprising finding in light of earlier perceptual studies showing that passive listening is usually not sufficient to yield reliable increases in activity in the parietal cortex (Zatorre et al., 2002; Warren and Griffiths, 2003). However, subsequent studies using a variant of the oddball paradigm have reported increased activity in IPL to changes in sound location relative to changes in sound identity even when the stimuli were not task-relevant and did not require overt motor responses (Altmann et al., 2007; Deouell et al., 2007). This discrepancy in the literature could partly be due to differences in experimental paradigms that emphasized the processing of location cues (Zatorre et al., 2002; Warren and Griffiths, 2003) rather than the representation of sound source location (Altmann et al., 2007; Deouell et al., 2007). 
The location-specific adaption in IPL provides further evidence that this region maintains a relatively detailed representation of the auditory space. Our findings extend those of prior studies using passive listening (Altmann et al., 2007; Deouell et al., 2007) by showing changes in neural adaptation for sound location even when the perceptual context does not involve infrequent changes in sound location. This is important because activity in IPL is modulated by auditory spatial attention (Shomstein and Yantis, 2006; Wu et al., 2007) and that infrequent changes in sound location may capture listeners' attention. The adaptation paradigm used in the present study is less likely to introduce attentional capture because it did not involve infrequent changes in sound location after relatively long stimulus repetitions. Indeed, in the present study half the trials comprised changes in sound location. Hence, the location-specific adaptation in IPL observed in Experiment 2 provides further evidence that this region maintains a representation of the auditory space. The changes in IPL activity may indicate a "mismatch" signal between representation of the sound source object and the new object location (Deouell et al., 2007). A mechanism that keeps track of location invariance could be particularly important for goaldirected action because changes in sound location may require a re-calibration of the efferent motor system such that appropriate motor responses (e.g., eye movement) could be generated to the new object location. The IPL may also act as an interface in which sound locations are remapped onto the visual co-ordinate system to guide motor responses. This hypothesis has received some support from a recent study using neuroelectric recordings which showed that preparation for responding to the location of a visual or an auditory target engages a similar neural network (Diaconescu et al., 2010). However, further research is needed to understand when and where spatial information from various sources (e.g., auditory and visual) are combined together.

There is increasing evidence from neuropsychological (e.g., Vallar et al., 1997; Baldo and Dronkers, 2006) and neuroimaging (e.g., Bledowski et al., 2006; Oztekin et al., 2009) studies indicating that the parietal cortex contributes to verbal working memory. In the present study, we used only three distinct virtual spatial locations $(-90,0,+90)$. Hence, it is possible that participants could have used the strategy to remember the words "left," "middle," and "right" and thus solve a verbal $n$-back task instead of an auditory spatial $n$-back task. Leung and Alain (submitted) recently investigated this possibility during an $n$-back $(n=1,2)$ working memory task for sound identity or location using the same materials as in Experiment 1. As expected accuracy and reaction times were worse for the 2-back than for the 1-back condition, albeit comparable in both "what" and "where" tasks. The analysis of fMRI data revealed an interaction between task and working memory load in the IPL. While increasing working memory load for sound identity yielded greater activation in the lateral and superior area of the right IPL, increasing working memory demand for sound location was paralleled by enhanced activity in the left supramarginal gyrus and the bilateral inferior and medial IPL. These specific changes in neural activity as a function of working memory load reveal domainspecificity within the parietal cortex.

Our findings and those of Leung and Alain (submitted) provide further evidence that the parietal cortex plays an important role in spatial working memory and are consistent with the neuropsychological literature. For instance, Carlesimo et al. (2001) found deficits in visuospatial working memory without impairment in verbal or working memory for shape in a patient with frontal and parietal lesions. Another case study also revealed deficits in $n$-back spatial working memory tasks without deficits in verbal working memory when deficits in visuospatial abilities were taken into account (Ravizza et al., 2005). With respect to audition, damage to dorsal brain regions including the parietal cortex often yields deficits in processing sound localization (Clarke et al., 2000, 2002; Bellmann et al., 2001; Adriani et al., 2003a,b). These findings suggest a selective deficit in remembering where sounds were located and may reflect deficits in encoding, maintaining, and/or updating spatial representations in working memory.

In the present study, increasing auditory spatial working memory load was associated with reduced BOLD effects in several brain areas thought to play an important role in identifying and remembering sound identity. This reduced activity in the medial and anterior portion of the temporal lobe during the 2-back minus the 1-back condition may indicate that sound identification was not performed as well when demand in processing and maintenance of spatial information in working memory was high. That is, as the demand in working memory increases for processing sound location, fewer resources are available resulting in reduced activity associated with the processing of sound identity. Evidence from scalp-recordings of event-related brain potentials has shown that working memory load modulates domain-specific activity (Alain et al., 2009) and may reduce processing of novel, irrelevant stimuli (SanMiguel et al., 2008). Evidence from fMRI studies also suggest that manipulation of working memory load may modulate domain-specific brain regions. For example, Strand et al. (2008) manipulated working memory demand for sound identity and found enhanced activity in the left inferior frontal gyrus and middle temporal gyrus during the maintenance phase of a phonological working memory task while the inferior parietal cortex was only active during the comparison and decision phase. Together, these findings suggest that distinct regions exist within the "what" and "where" pathways which are sensitive to working memory demand. The fact that varying working memory load for sound identity and location yields changes in the ventral and dorsal streams, respectively, provides strong support for the dual-pathway model, and reveals that neural networks supporting working memory performance are domain-specific.

The detection and response to infrequent repetition in sound location were accompanied by activity in a distributed set of regions that comprised motor cortex, IPL, superior parietal cortex, thalamus, and cerebellum. The activation in pre- and post-central gyri related to generating the response was not affected by working memory load. This suggests that activity in pre- and post-central gyrus index motor-related activity during response preparation and execution rather than stimulus-related processes, such as stimulusrepetition, which differed in the 1-back and 2-back tasks. The transient target-related activity in superior parietal cortex is consistent with previous studies showing increased activity in parietal cortex during target detection (e.g., Linden et al., 1999; Yoshiura et al., 1999; Kiehl and Liddle, 2001; Stevens et al., 2005). This responserelated activity in IPL is likely a part of a larger neural network involved in auditory-motor transformation. For instance, sounds 
that are associated with human action such as manipulated tools (Lewis et al., 2005) or material (Arnott et al., 2008) yield greater activation in IPL than other sounds (e.g., animal, human sounds). This IPL activity could be related to the retrieval of motor sequences associated with the production of the sounds (Lewis et al., 2005; Arnott et al., 2008).

In the present study, transient target-related activity in parietal cortices was little affected by working memory load, though a small increase in fMRI signal was observed in the right IPL when participants responded with their non-dominant hand (i.e., left hand). The peak activation was anterior and superior to that observed for sustained task-related activity (Experiment 1) and for taskirrelevant changes in sound location (Experiment 2). This effect may indicate increased attentional demands associated with using the non-dominant hand while comparing incoming sound location with a representation of the stimulus occurring two positions earlier. This interpretation corroborates previous work showing enhanced activity in inferior parietal cortex with increasing task demands (Blasi et al., 2007; Vohn et al., 2007), however, it is also possible that the target-related activity in IPL could reflect sensory effects associated with stimulus-repetition or low probability of repetition.

\section{REFERENCES}

Adriani, M., Bellmann, A., Meuli, R., Fornari, E., Frischknecht, R., Bindschaedler, C., Rivier, F., Thiran, J. P., Maeder, P., and Clarke, S. (2003a). Unilateral hemispheric lesions disrupt parallel processing within the contralateral intact hemisphere: an auditory fMRI study. Neuroimage 20(Suppl. 1), S66-S74.

Adriani, M., Maeder, P., Meuli, R., Thiran, A. B., Frischknecht, R., Villemure, J. G., Mayer, J., Annoni, J. M., Bogousslavsky, J., Fornari, E., Thiran, J. P., and Clarke, S. (2003b). Sound recognition and localization in man: specialized cortical networks and effects of acute circumscribed lesions. Exp. Brain Res. 153, 591-604.

Alain, C., Arnott, S. R., Hevenor, S., Graham, S., and Grady, C. L. (2001). "What" and "where" in the human auditory system. Proc. Natl. Acad. Sci. U.S.A. 98, 12301-12306.

Alain, C., He, Y., and Grady, C. (2008). The contribution of the inferior parietal lobe to auditory spatial working memory. J. Cogn. Neurosci. 20, 285-295.

Alain, C., McDonald, K. L., Kovacevic, N., and McIntosh, A. R. (2009). Spatiotemporal analysis of auditory "what" and "where" working memory. Cereb. Cortex 19, 305-314.

Altmann, C. F., Bledowski, C., Wibral, M., and Kaiser, J. (2007). Processing of location and pattern changes of natural sounds in the human auditory cortex. Neuroimage 35, 1192-1200.

Andersen, R. A., Snyder, L. H., Bradley, D. C., and Xing, J. (1997). Multiple representation of space in the posterior parietal cortex and its use in planning movements. Annu. Rev. Neurosci. 20, 303-330.

Argall, B. D., Saad, Z. S., and Beauchamp, M. S. (2006). Simplified intersubject averaging on the cortical surface using SUMA. Hum. Brain Mapp. 27, 14-27.

Arnott, S. R., Binns, M. A., Grady, C. L., and Alain, C. (2004). Assessing the auditory dual-pathway model in humans. Neuroimage 22, 401-408.

Arnott, S. R., Cant, J.S., Dutton, G. N., and Goodale, M. A. (2008). Crinkling and crumpling: an auditory fMRI study of material properties. Neuroimage 43, 368-378.

Arnott, S. R., Grady, C. L., Hevenor, S. J., Graham, S., and Alain, C. (2005). The functional organization of auditory working memory as revealed by fMRI. J. Cogn. Neurosci. 17, 819-831.

Baldo, J. V., and Dronkers, N. F. (2006). The role of inferior parietal and inferior frontal cortex in working memory. Neuropsychology 20, 529-538.

Belin, P., and Zatorre, R. J. (2003). Adaptation to speaker's voice in right anterior temporal lobe. Neuroreport 14, 2105-2109.

Bellmann, A., Meuli, R., and Clarke, S. (2001). Two types of auditory neglect. Brain 124, 676-687.

Blasi, G., Goldberg, T. E., Elvevag, B., Rasetti, R., Bertolino, A., Cohen, J., Alce, G., Zoltick, B., Weinberger, D. R., and Mattay, V.S. (2007). Differentiating allocation of resources and conflict detection within attentional control

In conclusion, the present results support the role of the dorsal stream in sound localization and suggest that the parietal cortex is important for monitoring and updating sound location in working memory regardless of whether or not a motor response is required or which hand is used to make the response. Although our findings suggest that memory-related activity for sound location can be dissociated from stimulus-response mapping and sensory-motor integration, further research using multiple levels of task difficulty and response demand is needed to better characterize such functional segregation. It is also important to better understand the role of IPL in processing and representing the spatial location of auditory and/or visual objects. For instance, does the IPL translate and/or integrate auditory and visual spatial co-ordinates into a common co-ordinate system (Cohen and Andersen, 2004). Further research may help identify the neural network involved in spatial processing and could clarify the role of the dorsal "where" pathways in binding spatial information from different sensory modalities.

\section{ACKNOWLEDGMENTS}

This research was supported by grants from the Canadian Institutes of Health Research and the Natural Sciences and Engineering Research Council of Canada.

processing. Eur. J. Neurosci. 25, 594-602.

Bledowski, C., Cohen Kadosh, K., Wibral, M., Rahm, B., Bittner, R. A., Hoechstetter, K., Scherg, M., Maurer, K., Goebel, R., and Linden, D. E. (2006). Mental chronometry of working memory retrieval: a combined functional magnetic resonance imaging and event-related potentials approach. J. Neurosci. 26, 821-829.

Buckner, R. L., Goodman, J., Burock, M., Rotte, M., Koutstaal, W., Schacter, D., Rosen, B., and Dale, A. M. (1998). Functional-anatomic correlates of object priming in humans revealed by rapid presentation event-related fMRI. Neuron 20, 285-296.

Carlesimo, G. A., Perri, R., Turriziani, P., Tomaiuolo, F., and Caltagirone, C. (2001). Remembering what but not where: independence of spatial and visual working memory in the human brain. Cortex 37, 519-534.

Clarke, S., Bellmann, A., Meuli, R.A., Assal, G., and Steck, A. J. (2000). Auditory agnosia and auditory spatial deficits following left hemispheric lesions: evidence for distinct processing pathways. Neuropsychologia 38, 797-807.

Clarke, S., Bellmann Thiran, A., Maeder, P., Adriani, M., Vernet, O., Regli, L., Cuisenaire, O., and Thiran, J. P. (2002). What and where in human audition: selective deficits following focal hemispheric lesions. Exp. Brain Res. 147, 8-15.

Cohen, Y. E. (2009). Multimodal activity in the parietal cortex. Hear. Res. 258, 100-105.
Cohen, Y. E., and Andersen, R. A. (2004). "Multimodal spatial representations in the primates parietal lobe," in Crossmodal Space and Crossmodal Attention, eds C. Spence and J. Driver (Oxford: Oxford University Press), 154-176.

Cox, R. W. (1996). AFNI: software for analysis and visualization of functional magnetic resonance neuroimages. Comput. Biomed. Res. 29, 162-173.

Deouell, L. Y., Heller, A. S., Malach, R., D'Esposito, M., and Knight, R. T. (2007). Cerebral responses to change in spatial location of unattended sounds. Neuron 55, 985-996.

Diaconescu,A.O.,Alain, C., and McIntosh,A. R. (2010). Modality-dependent "what" and "where" preparatory processes in auditory and visual systems. J. Cogn. Neurosci. doi: 10.1162/jocn.2010.21465 [Epub ahead of print].

Dosenbach, N. U., Visscher, K. M., Palmer, E. D., Miezin, F. M., Wenger, K. K., Kang, H. C., Burgund, E. D., Grimes, A. L., Schlaggar, B. L., and Petersen, S. E. (2006).A core system for the implementation of task sets. Neuron 50, 799-812.

Glover, G.H., Li, T.Q., and Ress, D. (2000). Image-based method for retrospective correction of physiological motion effects in fMRI: RETROICOR. Magn. Reson. Med. 44, 162-167.

Goodale, M. A. (2008). Action without perception in human vision. Cogn. Neuropsychol. 25, 891-919.

Hickok, G., and Poeppel, D. (2000). Towards a functional neuroanatomy of speech perception. Trends Cogn. Sci. 4, 131-138. 
Kiehl, K. A., and Liddle, P. F. (2001). An event-related functional magnetic resonance imaging study of an auditory oddball task in schizophrenia. Schizophr. Res. 48, 159-171.

Lewis, J. W., Brefczynski, J. A., Phinney, R. E., Janik, J. J., and DeYoe, E. A. (2005). Distinct cortical pathways for processing tool versus animal sounds. J. Neurosci. 25, 5148-5158.

Linden, D. E., Prvulovic, D., Formisano, E., Vollinger, M., Zanella, F. E., Goebel, R., and Dierks, T. (1999). The functional neuroanatomy of target detection: an fMRI study of visual and auditory oddball tasks. Cereb. Cortex 9 , 815-823.

Maeder, P. P., Meuli, R. A., Adriani, M., Bellmann, A., Fornari, E., Thiran, J. P., Pittet,A., and Clarke, S. (2001).Distinct pathways involved in sound recognition and localization: a human fMRI study. Neuroimage 14, 802-816.

Martinkauppi, S., Rama, P., Aronen, H. J., Korvenoja, A., and Carlson, S. (2000). Working memory of auditory localization. Cereb. Cortex 10, 889-898.

McLachlan, N., and Wilson, S. (2010). The central role of recognition in auditory perception: a neurobiological model. Psychol. Rev. 117, 175-196.

Ogawa, S., Lee, T. M., Kay, A. R., and Tank, D. W. (1990). Brain magnetic resonance imaging with contrast dependent on blood oxygenation. Proc. Natl. Acad. Sci. U.S.A. 87, 9868-9872.

Oztekin, I., McElree, B., Staresina, B. P., and Davachi, L. (2009). Working memory retrieval: contributions of the left prefrontal cortex, the left posterior parietal cortex, and the hippocampus. J. Cogn. Neurosci. 21, 581-593.

Poremba,A., Saunders, R. C., Crane,A.M., Cook, M., Sokoloff, L., and Mishkin, M. (2003). Functional mapping of the primate auditory system. Science 299, 568-572.
Rama, P., Poremba, A., Sala, J. B., Yee, L., Malloy, M., Mishkin, M., and Courtney, S. M. (2004). Dissociable functional cortical topographies for working memory maintenance of voice identity and location. Cereb. Cortex 14, 768-780.

Rauschecker, J. P. (1998). Parallel processing in the auditory cortex of primates. Audiol. Neurootol. 3, 86-103.

Rauschecker, J. P., and Scott, S. K. (2009). Maps and streams in the auditory cortex: nonhuman primates illuminate human speech processing. Nat. Neurosci. 12, 718-724.

Ravizza, S. M., Behrmann, M., and Fiez, J. A. (2005). Right parietal contributions to verbal working memory: spatial or executive? Neuropsychologia 43, 2057-2067.

Romanski, L. M., Tian, B., Fritz, J., Mishkin, M., Goldman-Rakic, P. S., and Rauschecker, J. P. (1999). Dual streams of auditory afferents target multiple domains in the primate prefrontal cortex. Nat. Neurosci. 2, 1131-1136.

SanMiguel, I., Corral, M. J., and Escera, C. (2008). When loading working memory reduces distraction: behavioral and electrophysiological evidence from an auditory-visual distraction paradigm. J. Cogn. Neurosci. 20, 1131-1145.

Scheibe, C., Wartenburger,I., Wustenberg, T., Kathmann, N., Villringer, A., and Heekeren, H. R. (2006). Neural correlates of the interaction between transient and sustained processes: a mixed blocked/event-related fMRI study. Hum. Brain Mapp. 27, 545-551.

Shomstein, S., and Yantis, S. (2006). Parietal cortex mediates voluntary control of spatial and nonspatial auditory attention. J. Neurosci. 26, 435-439.
Stevens, M. C., Calhoun, V.D., and Kiehl, K. A. (2005). fMRI in an oddball task: effects of target-to-target interval. Psychophysiology 42, 636-642.

Strand, F., Forssberg, H., Klingberg, T., and Norrelgen, F. (2008). Phonological working memory with auditory presentation of pseudo-words - an event related fMRI Study. Brain Res. 1212 48-54.

Uddin, L. Q., Supekar, K., Amin, H. Rykhlevskaia, E., Nguyen, D. A. Greicius, M.D., and Menon, V.(2010). Dissociable connectivity within human angular gyrus and intraparietal sulcus: evidence from functional and structural connectivity. Cereb. Cortex. 20, 2636-2646.

Vallar, G., Di Betta, A. M., and Silveri, M. C. (1997). The phonological shortterm store-rehearsal system: patterns of impairment and neural correlates. Neuropsychologia 35, 795-812.

Visscher, K. M., Miezin, F. M., Kelly, J. E., Buckner, R. L., Donaldson, D. I. McAvoy, M. P., Bhalodia, V. M., and Petersen, S. E. (2003). Mixed blocked/ event-related designs separate transient and sustained activity in fMRI. Neuroimage 19, 1694-1708.

Vohn, R., Fimm, B., Weber, J., Schnitker R., Thron, A., Spijkers, W., Willmes, K., and Sturm, W. (2007). Management of attentional resources in within-modal and cross-modal divided attention tasks: an fMRI study. Hum. Brain Mapp. 28, 1267-1275.

Warren, J. D., and Griffiths, T. D. (2003) Distinct mechanisms for processing spatial sequences and pitch sequences in the human auditory brain. $J$. Neurosci. 23, 5799-5804.

Warren, J. E., Wise, R. J., and Warren, J. D. (2005). Sounds do-able: auditorymotor transformations and the posterior temporal plane. Trends Neurosci. 28, 636-643.
Wenzel,E. M.,Arruda, M., Kistler, D. J., and Wightman, F. L. (1993). Localization using nonindividualized head-related transfer functions. J. Acoust. Soc. Am. 94, 111-123.

Wu, C. T., Weissman, D. H., Roberts, K. C. and Woldorff, M. G. (2007). The neural circuitry underlying the executive control of auditory spatial attention. Brain Res. 1134, 187-198.

Yoshiura, T.,Zhong, J.,Shibata, D. K., Kwok, W. E., Shrier, D. A., and Numaguchi, Y. (1999). Functional MRI study of auditory and visual oddball tasks. Neuroreport 10, 1683-1688.

Zatorre, R. J., Bouffard, M., Ahad, P., and Belin, P. (2002). Where is 'where' in the human auditory cortex? Nat. Neurosci. 5, 905-909.

Conflict of Interest Statement: The authors declare that the research was conducted in the absence of any commercial or financial relationships that could be construed as a potential conflict of interest.

Received: 15 June 2010; paperpending published: 27 June 2010; accepted: 27 October 2010; published online: 02 December 2010.

Citation: Alain C, Shen D, Yu H and Grady C (2010) Dissociable memory-and response-related activity in parietal cortex during auditory spatial working memory. Front. Psychology 1:202. doi: 10.3389/ fpsyg.2010.00202

This article was submitted to Frontiers in Auditory Cognitive Neuroscience, a specialty of Frontiers in Psychology. Copyright (C) 2010 Alain, Shen, Yu and Grady. This is an open-access article subject to an exclusive license agreement between the authors and the Frontiers Research Foundation, which permits unrestricted use, distribution, and reproduction in any medium, provided the original authors and source are credited. 